\title{
Arylethynyl Substituted 9,10-Anthraquinones: Tunable Stokes Shifts by Substitution and Solvent Polarity
}

\author{
Jinhua Yang1, Amala Dass' ${ }^{1}$, Abdel-Monem M. Rawashdeh ${ }^{1}$, Chariklia Sotiriou- \\ Leventis $^{1, *}$, Matthew J. Panzner ${ }^{2}$, Daniel S. Tyson ${ }^{3}$, James D. Kinder ${ }^{4}$, and Nicholas \\ Leventis ${ }^{4, *}$
}
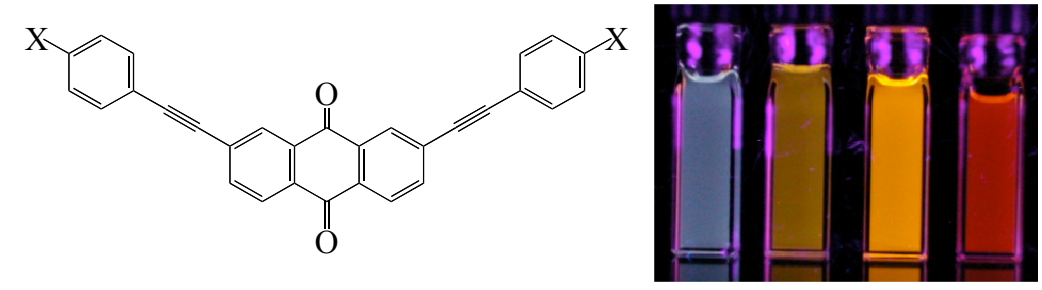

The title compounds are novel core-branch structures and demonstrate that long emission wavelengths $(>600 \mathrm{~nm})$ can be achieved through large Stokes shifts $(100-250 \mathrm{~nm})$ resulting from charge transfer transitions between branch-localized HOMO and a core-localized LUMO. The emission wavelength is fine-tuned by substitution. Strong photoluminescence from solid samples at $298 \mathrm{~K}$ is correlated to the crystal structure. 